ÉGYPTE monde arabe

\section{Égypte/Monde arabe}

$3 \mid 2000$

La censure ou comment la contourner

\title{
Le cinéma grand public égyptien entre censure et guichet
}

\section{Dina Galal}

\section{(2) OpenEdition}

\section{Journals}

Édition électronique

URL : https://journals.openedition.org/ema/797

DOI : 10.4000/ema.797

ISSN : 2090-7273

\section{Éditeur}

CEDEJ - Centre d'études et de documentation économiques juridiques et sociales

\section{Édition imprimée}

Date de publication : 30 juin 2000

Pagination : 105-124

ISBN : 2-87027-856-X

ISSN : 1110-5097

\section{Référence électronique}

Dina Galal, « Le cinéma grand public égyptien entre censure et guichet », Égypte/Monde arabe [En ligne], 3 | 2000, mis en ligne le 08 juillet 2008, consulté le 07 juillet 2022. URL : http://

journals.openedition.org/ema/797 ; DOI : https://doi.org/10.4000/ema.797

Ce document a été généré automatiquement le 7 juillet 2022.

Tous droits réservés 


\title{
Le cinéma grand public égyptien entre censure et guichet
}

\author{
Dina Galal
}

« L'art est une tentative de réponse à des questions portant essentiellement sur la révolte et la rébellion. » Oscar Ho Hong

1 L'histoire du cinéma montre bien qu'avec l'apparition de l'image animée, il y a un siècle, la nécessité s'est fait sentir simultanément d'imposer des restrictions à son mouvement, et ce dans le but de protéger «l'ordre public et les bonnes mœurs ». L'Égypte n'a pas échappé à la règle (Darwîsh, 1995, p. 91 ; Mumtâz, 1985, p. 31), qui a vu le cinéma éclore en son sein peu après la première projection cinématographique des frères Lumière à Paris (cAbd al-Basîr 1999, p. 61 ; Shafik, 1998, p. 10). Ce rapport de force entre le pouvoir et l'œuvre créatrice qui se poursuit depuis lors oppose tout un arsenal de lois et de règlements à des savoirfaire : si le pouvoir cherche à contrôler et façonner le courant créateur par ses procédures, ce dernier instrumentalise ses propres «techniques » afin de faire parvenir son message créatif (cAbd al-Fattâh, 1997). Dans le cas de l'Égypte, cette censure de toujours n'a pas pu pour autant endiguer le développement d'une véritable industrie, qui continue d'alimenter avec force le marché local et le marché arabe.

2 En jetant une lumière sur l'organisation et la logique internes de la censure sur le cinéma, mais aussi sur ses pratiques courantes et ses formes cumulatives, la présente étude cherche à dégager son impact direct et indirect sur les producteurs de films égyptiens dans les années quatre-vingt-dix et, plus largement, sur une industrie qui reproduit les conditions de sa permanence en épousant les contours de la conjoncture. Sans nier l'existence d'un autre cinéma, qualifié tour à tour de "parallèle » (mugâwir), de «différent » (mukhtalif), voire d'» exceptionnel»(istithnầî), on prendra ici pour objet - tout en se ménageant des points de comparaison historiques et généralistes - le cinéma grand public des années quatre-vingt-dix, communément appelé «courant prépondérant » ou « cinéma prédominant » ou encore « de masse ». S'il peut être défini 
commercialement en termes de box office, on verra que ce cinéma est en effet tout entier préoccupé et déterminé par les conditions de sa propre réception. On cherchera donc à voir selon quelles modalités la combinatoire de l'offre et de la demande, d'une part, et la censure, d'autre part, contribuent à former ce " courant » ou cette "vague » du cinéma grand public. Au-delà de la censure institutionnelle, des modalités de l'autocensure, des formes d'influence et d'intervention (informelle mais aussi officielle) exercées par des individus ou des groupes en vertu de leur position sociale, la présente contribution s'intéressera à la répercussion de tous ces facteurs sur les orientations tendancielles du cinéma comme sur les représentations liées à son patrimoine. Plus qu'aux intentionnalités, l'approche privilégiée ici s'attachera aux mécanismes, considérant donc ces formes comme des forces agissant sur la «réalité" cinématographique et déterminant ses traits.

La censure institutionnelle La censure sur les œuvres artistiques

Un dispositif figé. La Censure sur les arts et les imprimés (al-raqâba calâ al-funûn wa-lmatbûcât) a commencé en Égypte par "une réglementation des relations entre les “artistes" et le public : édit dans l'État moderne de Muhammad cAlî au début du XIX ${ }^{\mathrm{e}}$ siècle ; règlement de 1914, relatif aux imprimés et aux films, nécessité par la situation militaire et politique ainsi que par les affaires relatives à la sécurité publique suite au déclenchement de la première guerre mondiale. À l'époque, la censure relevait du ministère de l'Intérieur et était assumée par des étrangers qui veillaient aux intérêts des alliés. » En 1938, elle passe aux mains du ministère des Affaires sociales puis, en 1952, suite à la Révolution, à celui de l'Orientation nationale (wazârat al-irshâd al-qawmî) et enfin, au ministère de la Culture, dont elle relève jusqu'à nos jours, sous le nom de Censure sur les œuvres artistiques (al-raqâba calâ al-musannafât al-fanniyya) (Mumtâz, 1985, p. 9-25). La loi n 430 de 1955, «aussi stable qu'une montagne ", est la principale référence en matière de censure artistique, malgré les clauses modificatoires introduites par la nouvelle loi $\mathrm{n}^{\circ} 38$ de 1992 pour combler les lacunes dans des domaines nouveaux, notamment celui de la vidéo (Abû Shâdî, 1998, p. 36 ; Sharaf al-Dîn, 1992, p. 217). Il est important de signaler à ce propos les directives, non publiées, de 1947, émises par le ministère de la Culture et qui comportent deux listes : la première, propre au domaine social et moral, inclut 33 interdictions, la seconde, relative à la sécurité et à l'ordre public, en comprend 31 (Botiveau, 1993). Toutes ces directives ont été reconduites en vertu du décret du ministre de la Culture et de l'Information de 1976, concernant les « Principes de base de la censure sur les œuvres artistiques » dans lequel les interdits ont été "fondus » en une vingtaine d'interdictions (Darwîsh, 1995, p. 93-94). Le principal objectif de la loi sur la censure est le maintien de la sécurité et de l'ordre public, la protection des bonnes mœurs et des intérêts supérieurs de l'État.

Une discursivité variable. Malgré la relative stabilité de « l'esprit de la loi » régissant la censure à travers le temps, la terminologie utilisée aussi bien dans les déclarations que dans les écrits " varie », d'un censeur à l'autre, en fonction de l'époque (et donc, de l'idéologie dominante qui la sous-tend) et de la conjoncture. Ainsi, là où le censeur des années soixante évoque "la censure et son rôle dans la société socialiste ", insiste sur "le caractère impératif et nécessaire de la censure pour protéger la société des dérives ", et condamne "le film non socialiste » (Fawzî, 1997, p. 280-281), le censeur exerçant en l'an 2000 parlera du changement du rôle de la censure à l'aune des nouvelles technologies et des nouveaux médias (le satellite, internet), en insistant sur «la protection de la propriété intellectuelle des créateurs", «le soutien au véritable 
créateur quelle que soit sa vision ", ainsi que sur "la nécessité d'établir avec lui un dialogue sur les divergences de vue $»$. Ces variations discursives sont également liées à la personnalité et à l'origine socioprofessionnelle des censeurs.

5 La perception et l'accomplissement de leur rôle par ces divers censeurs nous sont accessibles à travers les autobiographies de censeurs professionnels qui présentent leur " témoignage » après s'être retirés du domaine, ainsi qu'à travers ce qui a été publié à propos de l'impact de la censure sur le cinéma grand public au moment où ils dirigeaient cette institution. D'un autre côté, un ensemble d'écrits critiques se rapportant essentiellement à la relation entre politique et cinéma, produits par des personnalités extérieures à l'organe de la censure (auteurs, critiques, chercheurs et professionnels des médias) nous renseigne précieusement sur la censure telle qu'elle est pratiquée par ces personnalités devenues censeurs par la suite.

6 Des censeurs pour une censure. Depuis que la censure relève du ministère de la Culture, la responsabilité a pu en incomber à des personnalités diverses provenant des milieux de la littérature, de la critique, du cinéma mais aussi de l'université. La fonction de censeur représente pour ces dernières une phase ascensionnelle et un volet transitoire de leur répertoire culturel et artistique qui, le plus souvent, s'est constitué au sein de l'institution officielle (c'est le cas, par exemple, de Mustafâ Darwîsh, Duriyya Sharaf al-Dîn, 'Alî Abû Shâdî, Madkûr Thâbit, etc.). En lisant certains de leurs écrits, publiés soit avant leur accession au poste de directeur de la censure, soit après qu'elles eurent abandonné cette fonction, nous avons pu constater que ce qui s'en dégage principalement est une critique générale de la fonction de la censure, menée du point de vue de l'intellectuel, du critique ou du créateur et non de celle du censeur. Si ces écrits ne rendent pas compte pour autant de ce qu'a été leur action en matière de censure, ni ne permettent de savoir dans quelle mesure cette action a été influencée par leur répertoire et leurs propres pratiques artistiques, il n'en reste pas moins que cette littérature peut - jusqu'à un certain degré - être considérée comme un témoignage sur les pratiques de censure. L'un de ces censeurs note que "les excès que recèlent les dispositions de la loi sur la censure ne l'ont pas surpris lorsqu'il fut nommé au poste de directeur ». Selon lui, « La loi ne traite pas le cinéma comme un art que ses créateurs peuvent exprimer librement, ou alors elle le traite en tant que tel mais avec beaucoup de réserves et de vigilance, voire de crainte. " (Darwîsh, 1995, p. 92) Se basant sur son expérience, il signale que ce qui renforce le caractère arbitraire de la loi et augmente les restrictions auxquelles est soumise la liberté d'expression cinématographique, réside autant dans l'interprétation de la loi que dans les pressions externes qui accablent le censeur: le climat de crispation intellectuelle, les considérations de relations publiques et l'intervention de personnes influentes (Darwîsh, 1995, p. 98). Un autre émet des réserves sur « le concept même de censure ", exprime son « espoir de la voir supprimée » et en appelle à « un cinéma différent, qui ferait face au cinéma grand public », « ce cinéma [à travers lequel] certains metteurs en scène créatifs essaient bien de décrire la réalité égyptienne avec sincérité et objectivité, mais qui reste un cinéma de consommation qui vit dans l'ombre du pouvoir (...), traite de la réalité avec vigilance, jouant sur un terrain sûr, sans prendre de risques, sans jouer avec le feu » (Abû Shâdî, 1998, p. 11-37).

7 Parmi les censeurs « de métier » qui ont gravi les échelons de l'administration même, deux femmes ayant occupé ce poste, l'une dans les années soixante-dix, l'autre dans les années quatre-vingt, en illustrent les pratiques de manière exemplaire. Pour étudier 
leur vision et leur expérience, nous nous sommes basée, pour la première, sur ses mémoires publiées, et pour la seconde, sur ce qui a été écrit au sujet de son action. Les Mémoires d'un censeur raconte l'expérience de la première femme ayant occupé le poste de directeur général de la censure sur les films, puis, sur les œuvres artistiques en général. Ittidâl Mumtâz y évoque «les efforts qu'elle a déployés pour apprendre aux autres le respect [de l'activité de censure]» dans un climat d'incompréhension où d'aucuns ont pu la traiter de " ciseaux tranchants » (Mumtâz, 1985, p. 6). Une question de civilisation, la censure sur les œuvres artistiques, loin de se limiter aux seules pages de son livre, fera l'objet d'un débat tant qu'il y aura de la littérature et de l'art et tant qu'il y aura des sociétés (Mumtâz, 1985, p. 25). Un chapitre entier, qui s'ouvre par le récit de ses désillusions, est consacré à la "résistance des institutions artistiques relevant du ministère de la Culture aux lois sur la censure ", et ce alors même que ces dernières "dépendent toutes d'un même ministère ». En l'absence de coordination et de travail en bonne intelligence, l'auteur relate ses difficultés à tenir les rênes de la censure, « ces institutions estimant en effet qu'elles sont en mesure de pratiquer ellesmêmes une autocensure, indépendamment de la Direction centrale, dont l'autorisation ne constitue guère plus qu'une formalité " (Mumtâz, 1985, p. 237). Son évocation implicite du précédent qu'a constitué la censure de sa censure est d'un intérêt plus direct pour nous: le passage où elle décrit son activité comme «un chemin semé d'embûches... faisant plusieurs victimes parmi ceux qui, paradoxalement, regardent pourtant de près où ils mettent les pieds » (Mumtâz, 1985, p. 6), fait en effet allusion à la sanction subie par la direction de la Censure sur les œuvres artistiques en 1976, deux mois après la projection du film de Saî̀d Marzûq, al-Mudhnibûn (Les fautifs). Représentant l'Égypte au Festival du film du Caire, ce film socio-politique, tiré d'un roman de Naguib Mahfouz, devait amener la directrice de la censure ainsi qu'un certain nombre de ses collaborateurs à passer en conseil de discipline pour avoir autorisé sa projection et son exportation (Sharaf al-Dîn, 1992, p. 225-226). «Le dispositif de la censure en fut marqué et depuis, les censeurs tremblent. " (Dardîr, 2000) Selon les attendus de l'enquête, le film comporte des contraventions criantes touchant aux mœurs publiques, au "secteur public», aux "valeurs religieuses et morales de la société ", " favorisant le vice » et constituant « un appel explicite à la propagation de la débauche » (Sharaf al-Dîn, 1992, p. 225). Bien que l'affaire - qui dura quatre ans - se soit soldée par des amendes ou des déductions salariales, la simple décision de l'instruire fut perçue par tous ceux qui travaillaient dans cette instance comme un avertissement, les intimant à user au maximum de leur pouvoir d'interdiction, de suppression et d'annulation afin d'éviter les sanctions et d'être maintenus dans leurs fonctions. Ce fut aussi un message clair et menaçant adressé aux cinéastes: leurs œuvres futures pourraient ne jamais voir le jour au cas où ils dépasseraient la ligne rouge tracée par ce précédent (Sharaf al-Dîn, 1992, p. 225 ; Salâh al-Dîn, 1996, p. 209-229). Notre deuxième exemple, celui de Nacima Hamdî, illustre de manière encore plus évidente ces pressions extérieures qui poussent la censure dans les retranchements d'une surenchère anticipatrice : «L'administration chargée de la censure a contribué, sous la présidence de cette directrice, à la consécration des objectifs des groupes radicalisés qui ont influencé l'opinion publique de l'époque, avant que l'État n'intensifie sa lutte contre eux et ne permette aux cinéastes de les approcher, voire de les attaquer. " (Abû Shâdî, 1997, p. 133-136). Considérant et déclarant que «toute violation des mœurs dans l'art équivaut à leur violation dans la vie », Nacîma Hamdî fut par suite surnommée la «dame de fer " de la censure (Abû Shâdî, 1997, p. 134). Moins ultra, son successeur 
devait tenter d'expliquer sa position en ces termes : «Elle était personnellement déjà conservatrice. Sans doute n'avait-elle pas pour but conscient de renforcer l'extrémisme militant, mais elle était focalisée sur les questions de mœurs. » (Abû Shâdî, 1997, p. 134) C'est d'ailleurs à cette même époque que la mention "Pour adultes seulement" disparut des affiches de films comportant des scènes qu'il était préférable de ne pas exposer aux mineurs : contrairement à l'usage dans la plupart des pays, la directrice de la censure tenait à ce que toute œuvre artistique convienne à tous les âges (Abû Shâdî, 1997, p. 134). Aujourd'hui, les films sont à nouveau classés en deux catégories : les "films pour tous", et les "films pour adultes seulement" (Karkûtî, 1999), ce qui n'empêche pas ces derniers de passer par les ciseaux de la censure.

8 Censurer ou façonner le cinéma ? Si, à court terme, la fonction précise de la censure sur les films se limite à censurer ceux qu'elle reçoit et non à orienter la production cinématographique, à plus long terme, cette affirmation n'est pas rigoureusement exacte. Les études critiques portant sur les relations du cinéma et de la politique durant la deuxième moitié des années quatre-vingt relèvent des cas de « rejets de scénarios, changements de contenu, suppressions de personnages, modifications du dénouement." Cet interventionnisme devait entraîner «l'imposition d'une autocensure très sévère chez les cinéastes dont les répercussions se ressentirent sur la structuration du marché du film » (Abû Shâdî, 1998, p. 135). C'est ainsi que des films strictement commerciaux, tournés à la hâte avec des budgets restreints virent le jour. D'un niveau médiocre pour la plupart, ces «films d'entrepreneurs ", ainsi surnommés, ne flirtent avec aucun interdit. L'année 1986 connut à ce titre une production record dans l'histoire du cinéma égyptien : 95 films autorisés par la directrice de la censure furent projetés (Abû Shâdî, 1998, p. 134).

9 Le dénouement à l'épreuve des ciseaux. Parmi les formes d'interventionnisme les plus aiguës et les plus récurentes dans l'histoire du cinéma égyptien, la modification du dénouement figure en bonne place. Des années trente jusqu'aux années quatre-vingt, on ne compte plus les films concernés par ce type d'interventionnisme et les films Lâshîn de Fritz Kramp et al-Barî' (L'innocent) de 'Âtif al-Tayyib, en sont, chacun à son époque, exemplaires. L'une des premières productions des Studios Misr, réalisée dans le cadre du programme économique national de Talcat Harb, Lâshîn, est un film historique qui traite de la corruption d'un gouvernant au temps des Mamelouks. Fait exceptionel et qui ne se reproduisit jamais en Égypte, le film fut interdit le jour même de sa première (17 mars 1928), « dans l'intérêt public et sa primauté par rapport à l'intérêt particulier " et ressortait en salle en novembre de la même année, mais sans son dénouement initial: jugé subversif, ce dernier avait été remplacé par une fin plus conciliante. Ce film qui avait eu le courage d'établir un lien entre la corruption politique et le déclin économique avait été jugé intolérable par la démocratie des années trente avec ses partis et son Parlement (Abû Shâdî, 1998, p. 182 ; Salâh al-Dîn, 1996, p. 4564 ; 'Abdû, 1999). Quant à L'innocent (1986), qui avait prédit la mutinerie des conscrits de la sécurité centrale de février de la même année, il fit l'objet de la plus grande coupure de l'histoire du cinéma égyptien (environ 20 minutes) en même temps que son dénouement était également modifié. Une commission ministérielle supérieure regroupant les ministres de la Défense, de l'Intérieur et de la Culture visionna le film et jugea qu'il était " plus subversif que simplement divulgateur » (Abû Shâdî, 1998, p. 191; Dardîr, 2000 ; Salâh al-Dîn, 1996, p. 31, 250). Bien que la version originale ait été projetée à l'étranger et ait recueilli des prix dans des festivals internationaux, ce film, 
également primé localement, ne fut finalement projeté en Égypte que dans une version tronquée et amputée de sa fin. Il y a donc eu deux versions différentes : l'une pour le marché local, l'autre destinée à l'exportation (Salah al-Dîn, 1996, p. 249 ; Shafik, 1998, p. 36). Le réalisateur lésé saluait néanmoins le courage des censeurs qui avaient "admiré le film en tant que citoyens, et manifesté leur sympathie en autorisant sa projection "... jugeant que «les coupures avaient été faites sous l'influence de la commission ministérielle, en coordination avec les producteurs du film » qui avaient refait leurs calculs.

La censure et ses thuriféraires. La justification de la censure par ses censeurs et thuriféraires, qui n'est pas à une incohérence près, fait par suite feu de tout bois, puisqu'elle ne craint pas, au prix de sa logique interne, de s'appuyer sur le sophisme et l'analogie malheureuse. Cet "art de couper " ne serait finalement décrié que par les mauvais cinéates, qui s'en emparent comme d'un «prétexte, alors que le bon cinéaste peut toujours trouver le moyen de s'exprimer ». À ce titre, on a pu dire que c'est parce que le cinéma iranien était soumis à une censure analogue à celle que connaissent les films égyptiens que ce cinéma connaissait une consécration internationale aujourd'hui. Sans s'attarder sur les raisons de l'absence d'une telle consécration pour le cinéma égyptien contemporain, on avance que le cinéma iranien contemporain serait parvenu à percer mondialement avec des films d'une grande sensibilité artistique sans pour cela tomber dans les interdits du sexe, de la violence et de la politique. De ces interdits innombrables dont il tirerait sa gloire, la revue al-Fann al-sâbic (Le septième art) qui consacre un numéro au cinéma iranien', fait état, rendant compte du travail et des efforts considérables consentis par les réalisateurs, devenus « maîtres » dans l'art de la métaphore, du message codé et du contournement des interdits, afin de passer entre les mailles de la censure. Signalons la suppression récente de cette dernière en Iran, qui rend désormais l'artiste responsable devant « la société » et « la justice ».

11 Ces incohérences de la censure citées plus haut sont aussi bien sûr à mettre sur le compte des conditions de sa production. Difficiles et indigentes depuis toujours, ces dernières ne sont pas niées par la direction de la censure actuelle'. Si les conditions de travail (bas salaires, lieux inappropriés) expliquent aussi bien la fuite des compétences que la corruption caractérisée (Darwîsh, 1999), la médiocrité de la culture artistique de la majorité des censeurs qui - à l'exception de certains diplômés de l'Académie des arts - sont des fonctionnaires pour la plupart, éclairent les choix imprévisibles, fantasques, de ses cisailleurs.

La télévision contre le cinéma ou la métacensure

12 La télévision, cet " ogre » ou ce " monstre » qui envahit les maisons sans y être invité et qui est accueilli par tous indépendamment de l'âge ou du «niveau de compréhension » requis, aurait de quoi justifier le besoin d'un « censeur particulier » qui passe au crible les films dont la Censure sur les œuvres artistiques a déjà autorisé la projection commerciale en salle et qui les marque de son empreinte particulière avant leur diffusion sur le petit écran. Ainsi, la censure pratiquée par la télévision sur les films qu'elle diffuse est une censure après censure, un acte ajouté à la dramaturgie de la censure. La multiplicité de ses instances aggrave ainsi un dispositif déjà lourd en soi par ses effets cumulatifs.

«Cri de détresse des réalisateurs : qui va sauver nos films de la censure télévisuelle?» titrait le périodique spécialisé al-Fann al-sâbic sur la couverture de son numéro de novembre 1999. Des cinéastes (scénaristes, distributeurs et producteurs) y exposent les 
pratiques auxquelles leurs films sont soumis lorsqu'ils doivent être diffusés sur les chaînes nationales de télévision - toutes publiques. Suppression, amputation, atteinte au contenu, à la teneur intellectuelle et artistique: ces qualifications auxquelles recourent les cinéastes lésés sont particulièrement parlantes quand on sait que ces pratiques sont menées à l'insu des intéressés (Hasan, 1999). Ces faits accomplis sont tels que certains cinéastes, tels Youssef Chahine, en sont à demander à s'occuper euxmêmes du montage après suppression des séquences de leurs films destinés à la diffusion télévisée, afin qu'ils ne soient pas mutilés ('Abd al-Basîr, 1999, p. 65).

Effets cumulatifs, avons-nous dit. Effet pervers, aussi : la censure télévisuelle exerce de fait une tutelle sur la direction de la Censure sur les œuvres artistiques qui, du coup, anticipe sur les réquisits de la censure télévisuelle avant d'autoriser la projection en salle des films qui lui sont soumis (Hasan, 1999, p. 10-11). Il n'est pas jusqu'à la directrice de la Censure sur les œuvres artistiques qui ne donne raison à la censure télévisuelle: "Une fois que la télévision a acheté le film, la version acquise est comparable à une robe qu'il appartient à l'acheteuse d'élargir ou de rétrécir à son gré sans que le commerçant ne puisse s'y opposer puisqu'il l'a vendue et qu'il en a touché le prix... Le réalisateur qui craint que son film soit mutilé n'a devant lui qu'une seule solution, celle de recourir à la justice. » (Hasan, 1999, p. 10-11). Plus qu'une confusion entre propriété intellectuelle et biens de consommation, c'est d'une confusion entre loi sur la censure et loi du marché dont il s'agit ici : "aux réalisateurs de se plier aux exigences des chaînes de télévision pour pouvoir vendre leurs œuvres, sinon, qu'ils subissent les conséquences de leurs décisions» (Hasan, 1999, p. 10-11). Dans quelle mesure ces propos d'un distributeur reflètent-ils une création cinématographique orchestrée «selon les normes du marché télévisuel»? Les lignes suivantes pourront peut-être apporter quelques éléments de réponse.

15 C'est dans le contexte de la crise générale de l'industrie du cinéma, de sa privatisation inéluctable et de la restructuration de ses activités, que survient l'invasion par la télévision et les institutions qui en relèvent du champ de la production cinématographique. Le système producteur/réalisateur (Galal, à paraître), dans lequel la télévision joue le rôle du mécène des films réalisés par des cinéastes confirmés, est une configuration répandue dans beaucoup de pays et est favorablement accueillie par les critiques locaux du fait qu'elle contribue à résoudre la crise du cinéma. Le cahier des charges, cela dit, est contraignant : pour qu'un film soit financé par la télévision, il ne doit contenir ni mythe, ni «extravagance» et ne doit pas s'adresser aux sens (alKardûsî, 1999, p. 234). In fine, il s'agit, pour la télévision, de "présenter un cinéma sérieux, qui se refuse à flirter avec le guichet à la manière des films grand public » (Hilmî, 1998). Cette expérience a soulevé - quant au principe - un ensemble de protestations. Selon certains, la télévision mène « une expérimentation dans un champ qui n'est pas le sien et conformément aux mécanismes télévisuels et de censure qui lui sont propres ». Comment, en effet, «soumettre le film cinématographique aux critères de la censure télévisuelle sans dénaturer ce qui constitue le propre d'un scénario cinématographique, son langage particulier, le traitement du sujet, des événements et des séquences?» ('Abd al-Basîr, 1999). D'autres contestent encore l'interventionnisme télévisuel, comme ce réalisateur qui se plaint de ce que des séquences entières de l'un de ses films, financé par l'Union de la télévision, ont été supprimées lors de sa projection au Festival national du cinéma en 1999, « portant préjudice à son contenu et à sa teneur artistique et intellectuelle (...), ce qui influença la décision du jury » (Hasan, 1999). Afin de surmonter ce problème et de libérer le cinéma financé par la télévision 
du pouvoir de censure de cette dernière, un réalisateur propose "que le réalisateur remette deux versions du même film: l'une destinée à la projection en salle et l'autre soumise à la censure de la télévision et à ses conditions, quitte à devoir tourner deux fois certaines séquences impropres à la diffusion télévisuelle et à dépenser ainsi plus de temps et d'argent» ('Abd al-Basîr, 1999). Cette proposition est aujourd'hui sérieusement à l'étude...

Les artistes contre l'art ou l'autocensure « négatrice »

Doublant ou précédant cette double censure institutionnelle, l'«autocensure négatrice » va jusqu'à trouver chez les gens du métier eux-mêmes de puissants relais. S'il concerne, dans ses formes les plus spectaculaires, des cas isolés, ce phénomène qui voit se dresser des artistes contre leur art, ne peut être compris dans sa globalité si on ne le rattache pas au rôle joué par la salafiyya al-mutashaddida, ce traditionalisme ambiant intransigeant, que d'aucuns ont pu décrire comme ce qui, dans le cinéma contemporain, «entrave le plus l'œuvre créatrice (...) puisqu'il nourrit une hostilité générale à l'égard de toute forme de créativité artistique, et en particulier à l'égard du cinéma, considéré comme la forme la plus éloquente et la plus influente de cette dernière » ('Abd al-Basîr, 1999). Par autocensure négatrice, on entend ainsi l'ensemble des opinions et des pratiques individuelles observables des cinéastes, relatives à la détraction du septième art et qui s'expriment en fonction de critères extra-artistiques. Parce qu'elle influence sûrement, quoique dans une mesure difficilement appréciable, le style de la production cinématographique, la reproduction des conditions nécessaires à son évolution (ou à sa continuité), les représentations liées à son patrimoine et, parfois, les moyens de conservation de sa mémoire, il nous a paru pertinent d'évoquer ici cette entreprise de sabordement - dont il est utile de rappeler qu'elle ne prend pas d'abord sa source dans une intériorisation d'une censure officielle bien comprise mais bien plutôt dans l'intériorisation des diktats d'une censure morale diffuse, ellemême ordonnatrice pour partie de la censure officielle, comme illustré plus haut.

De telles pratiques, qui vont, dans les cas extrêmes, jusqu'à l'auto-exclusion ou « l'exil volontaire » hors du champ du cinéma pour les industriels et les artistes de ce secteur, comprennent aussi bien le refus ou le choix de rôles déterminés par les acteurs, la suppression de certaines scènes au moment du tournage, préalablement classées en scènes « convenables " et "non convenables ", qu'une surenchère dans le discours de certains réalisateurs qui se vantent de produire des films " propres » et conservateurs, indépendamment de la pauvreté du contenu dramatique et du langage cinématographique (Abû Shâdî, 1998, p. 132 ; al-Cinema wa-l-nâs, 1998; Hilmî, 1998; Qatâmish, 1998 ; Sacad, 1998 ; 'Abd al-Basîr, 1999, p. 66). Parmi les cas d'auto-exclusion, les plus retentissants et sans doute les plus délétères pour l'industrie cinématographique sont ceux qui s'accompagnent d'une remise en cause du principe même de la pratique de cet art que d'aucuns rejettent pour des motifs dogmatiques (muntalaqât caqấ'idiyya). C'est ainsi que la scène cinématographique égyptienne a connu récemment des désertions successives de plusieurs actrices qui, non contentes d'abandonner le métier, se sont lancées, pour certaines d'entre elles, dans sa stigmatisation et dans un prosélytisme à l'adresse de leurs confrères - et surtout consœurs - restés fidèles au septième art. Certains films ont même été retirés du marché de la vidéo à la demande de l'une de leurs interprètes repentie qui a en outre exigé d'en interdire la projection à l'échelle commerciale. 

suite, une atteinte directe au patrimoine cinématographique du pays. La confusion qui règne entre patrimoine et propriété "matérielle» a suscité de vifs débats dans la presse spécialisée. Ainsi, l'hebdomadaire égyptien al-Musawwar publie, dans deux numéros successifs, deux articles intitulés : «Hal min haqq alwaratha icdâm acmâl almubdic? " (Les héritiers ont-ils le droit de détruire les œuvres des créateurs ?) et " Abnâ" Husayn Sidqî ahraqû aflâmuhu " (Les enfants de Husayn Sidqî ont brûlé ses films) (Sacad, 1998a et 1998b). Les deux articles rapportent que les enfants de cet illustre acteur de cinéma des années trente et quarante ont brûlé les négatifs des meilleurs films de cet acteur/producteur (quatorze films) « exécutant ainsi les dernières volontés d'un homme blessé qui avait jugé insuffisant l'hommage rendu à ses œuvres ». Producteur pionnier dans les années quarante, cet artiste engagé politiquement et socialement, devait se voir confisquer plusieurs films de son répertoire qui en comptait environ soixante-dix. Nommé membre de l'Assemblée nationale (majlis al-umma) à la suite de la révolution de juillet 1952, il se retire de la scène cinématographique en 1962 et meurt en 1976. D'après les propos tenus par l'un de ses fils, il aurait, «en quelque sorte », douté de la légitimité, sur le plan idéologique, du métier d'artiste qu'il avait exercé tout au long de sa vie (Sacad, 1998a) - ce qui justifierait aujourd'hui cet acte de prévarication.

Censure politique, censure publique

19 Autocensure et censure institutionnelle ne constituent par suite que la partie visible de l'iceberg. D'autre formes d'intervention et d'influence, perceptibles au sommet comme à la base, font que tout le monde est censeur, à un titre ou à un autre : depuis ceux qui sont à la tête du régime et se substituent au censeur parfois, jusqu'à l'homme de la rue, en passant par les courants, les groupes et les catégories sociales qui exercent diverses formes de censure où l'art est confondu avec la réalité. Dans les années quatre-vingt-dix, alors que certaines de ces formes marquent un retrait, d'autres resurgissent et on voit apparaître des pratiques nouvelles. Pour schématiser à très gros traits, on pourrait dire que si le cinéma des années soixante et soixante-dix est souvent la victime d'une censure politique, celui des années quatre-vingt et quatre-vingt-dix est l'otage de l'espace public - que celui-ci se targue de défendre des intérêts corporatistes ou des «valeurs », morales ou religieuses.

Le cinéma des années soixante et soixante-dix, que caractérise la prudence, a ainsi connu des formes intransigeantes de censure et a fait les frais de conflits entre la censure et les éléments conservateurs du pouvoir. Étaient particulièrement touchés les films tirés d'œuvres littéraires (plusieurs romans de Naguib Mahfouz, Tharwat Abâza) comportant une critique politique directe ou indirecte. Dans certains cas, l'intervention du président de la République en personne était nécessaire, pour décider s'il s'estimait visé ou menacé par ces films et si ces derniers menaçaient la patrie: "Nasser a dû intervenir lui-même, précisément en 1969, et jouer le rôle de censeur en visionnant les films litigieux qui, aux yeux de ses collaborateurs, portaient atteinte à sa personne ou à la Révolution ou qui soutenaient un courant adverse ", prouvant, lorsqu'il prenait le contre-pied de «certains responsables prudents et circonspects, que le régime et son chef avaient des vues plus ouvertes que les leurs » (Abû Shâdî, 1998, p. 169; Dardîr, 2000).

21 Bien que le cinéma égyptien des années quatre-vingt soit également et «dans l'ensemble, docile et apprivoisé, il ne manque pas pour autant de soulever de temps à 
autre quelques remous qui inquiètent le gouvernement, causent une tension au niveau de la censure, provoquent l'opinion publique jusqu'à entraîner la comparution des artistes devant les tribunaux ou leur arrestation, le versement d'une amende ou l'interdiction de leur produit " (Abû Shâdî, 1998, p. 159). La censure informelle ici prenait souvent la forme organisée d'une protestation corporatiste qui aboutissait à saisir la justice. Ainsi de l'action intentée, en 1984, par quelques avocats contre le film de Ra'fat al-Mîhî, L'avocat, qui, selon eux, portait préjudice à la profession et dont ils demandaient l'interdiction. Le producteur, le réalisateur et les deux acteurs interprétant les rôles de l'avocat et du juge furent condamnés à un an de prison, mais l'affaire se conclut par voie de conciliation et de compromis, et par des excuses. De même, des boulangers firent un esclandre lors de la sortie du film al-Furn (La boulangerie) dont le héros est un boulanger impliqué dans un trafic de drogue, ce qui aurait porté "préjudice à la profession" (Abû Shâdî, 1998, p. 135-167). Ce type de « censure » corporatiste était parfois contré par une action également corporatiste : en 1986, grâce à leur solidarité, intellectuels, critiques et journalistes ont réussi à sauver les producteurs du film Li-l-hobb qissa akhîra (Une ultime histoire d'amour, réalisé par Ra'fat al-Mîhî) et à arrêter l'action en justice menée contre les acteurs, accusés d'outrage à la pudeur - acte qualifié à l'époque de "comportement scandaleux en studio ». Il n'est pas jusqu'à des individus qui aient intenté des actions en justice, contribuant, même en cas de rejet de leurs plaintes, à alourdir le climat déjà contraignant dans lequel évoluent les créateurs: ainsi d'une plainte déposée par le maire (cumda) d'un village contre le film al-Mutasawwil (Le mendiant) au motif que le village mentionné dans le film porte le même nom que le sien, nuisant de ce fait à sa réputation.

Les années quatre-vingt-dix ont, quant à elles, connu une véritable escalade judiciaire où l'objet du litige est moins l'atteinte portée aux intérêts particuliers de groupes, de métiers ou d'individus que celle portée aux intérêts généraux de la morale et de la religion : ainsi de la multiplication de ces requêtes en hisba (action en préservation des intérêts islamiques), dont le propre, jusqu'à ce que l'État n'en restreigne la portée et les modalités légales, résidait dans le fait qu'elles pouvaient être motivées et adressées par des individus. Sont touchés des films récents comme des rediffusions. À titre d'exemple, citons ici les cas de requête en hisba contre l'affiche du film de Husayn Kamâl, Abî fawq al-shagara (Mon père sur un arbre perché), film autorisé et diffusé avec la même affiche il y a plus de vingt-cinq ans et rediffusé récemment. La reproduction, en couverture de la revue al-Cinema wa-l-nâs (dans sa livraison du 20/03/1999), d'une scène du film de Sharîf 'Arafa Tuyûr al-zalâm (Les oiseaux de l'ombre), sans entraîner de requête en hisba, fut, quant à elle, attaquée pour « atteinte à la pudeur du public » (Abû Shâdî, 1998, p. 133 ; Dardîr, 2000). En 1994, c'est au tour d'al-Muhâjir (L'émigré) de Youssef Chahine, pourtant autorisé et diffusé localement et à l'étranger, de voir sa projection interrompue (Bernard-Maugiron, 1997). « Les poursuites judiciaires au nom de la hisba, les plaidoiries et les témoignages jetés sur la place publique, les articles et les déclarations publiés dans la presse comportant des menaces indirectes contre toute une catégorie de citoyens ", signifient, au fond, « un refus absolu de la créativité » et conduisent à une sorte de «désertion du terrain de la créativité » (Soueif, 2000, p. 243-250).

Le cinéma grand public ou la réception à tout prix 

cinéastes s'organise-t-elle en contrepartie? Quels fruits les germes de leur créativité donnent-ils sur ce «terreau»? On a vu comment les différentes formes de la censure institutionnelle font qu'un même film, dans sa version définitive, est traité comme un tissu élastique ou comme une matière souple et malléable. Ce traitement et la riposte qu'il entraîne de la part des gens du métier a permis la ramification inattendue du marché. Parallèlement à la version originale, il en existe autant de versions qui vont différer, en fonction du moment de la censure (refus de ce qui a précédemment été autorisé et autorisation de ce qui avait été refusé totalement ou partiellement), du cadre de sa projection, du marché et des "appréciations » du censeur. On trouvera donc une version destinée à la commercialisation locale et à la diffusion en salle, une autre pour le marché de la vidéo à usage personnel ou collectif, une autre encore destinée à la diffusion télévisée sur les chaînes locales, une autre pour la diffusion par satellite et enfin une version pour la présentation du film et sa commercialisation à l'étranger (festivals, pays du Golfe et Europe en particulier, pour les coproductions).

La question qui se pose alors est celle de la continuité de l'industrie du cinéma sur le plan de sa créativité et de sa commercialisation - indépendamment de ses crises et des problèmes généraux et périodiques qui accompagnent cette "continuité " - dans le contexte d'une censure qui, loin de régresser, ne fait que se ramifier, se réajuster et se diversifier aussi bien dans ses formes, ses sources que dans ses logiques. Accompagnant le cinéma égyptien depuis son apparition, la censure touche tous les genres mais aussi tous les courants ('Abd al-Basîr, 1999 ; Shafik, 1998) : le cinéma grand public des années quatre-vingt-dix, dont la prospérité n'est plus à démontrer, échappe-t-il à son action et selon quels procédés? Simple réponse du berger à la bergère? mais alors comment expliquer qu'il constitue un véritable " phénomène de société »?

Regroupant les films qui sont restés le plus longtemps à l'affiche (un an en moyenne), qui passent simultanément dans le plus grand nombre de salles - dans la capitale aussi bien qu'en province - et qui enregistrent le plus grand nombre de spectateurs (jusqu'à près de quatre millions, selon la presse, pour Sacîlî fîl-gâmica al-amrîkiyya), ce cinéma devait surprendre la critique par l'ampleur de son succès. Partisans ou détracteurs, tous s'interrogent, à divers degrés, sur les causes de cette « connivence entre le public et le cinéma prédominant", sur la crise née de l'opposition "entre la créativité cinématographique sérieuse et la culture du récepteur " (Saîd, 1999). Le grand succès de ses débuts a même surpris ses producteurs et a dépassé toutes leurs espérances, les poussant en conséquence à produire des "séries" de films sur la base d'un même canevas, avec quasiment le même casting et la même équipe derrière la caméra (Zuhayrî, 1999). Pourquoi le cinéma prédominant des années quatre-vingt-dix a-t-il pris cette tournure superficielle alors même qu'on a pu enregistrer à cette époque un assouplissement relatif de la censure officielle? Pourquoi ce type de films a-t-il été si favorablement accueilli par le public? Selon quels termes de l'équation «censure/ guichet » le cinéma prédominant des années quatre-vingt-dix a-t-il été façonné ?

Bien que ce cinéma appartienne, de manière générale et conformément aux normes de classification américaines, au genre comique, et que ce type de comédie serve à exprimer la réalité politique et sociale de l'Égypte des années quatre-vingt-dix (al'Amrî, 1999, p. 75), il n'en reste pas moins que l'on peut y distinguer deux genres liés à deux conjonctures, l'une politique, l'autre sociale.

Égypte/Monde arabe, 3 | 2000 
27 La première concerne surtout la première moitié des années quatre-vingt-dix, même si elle se prolonge, à un moindre degré, pendant la seconde moitié de la décennie. Les films grand public produits alors combattent deux phénomènes : la corruption mais aussi le terrorisme, dans le cadre de la lutte anti-terroriste engagée par l'État contre les gamâcât, ces groupements qui imposent leurs opinions par les armes (Abû Shâdî, 1998, p. 136-137). La série de films de cÂdil Imâm, star engagée dans la lutte contre le terrorisme (égyptien mais aussi algérien) et la corruption - aussi bien d'ailleurs dans les films que dans les pièces de théâtre qu'il interprète -, concorde avec les orientations du gouvernement et reçoit " un accueil calculé que nul ne saurait nier » (Ramsîs, 1999, p. 97). Ici donc, les calculs de l'État prévalent sur le pouvoir du censeur, constituant, en quelque sorte, une censure par le haut, mais la célébrité de l'acteur-interprète, qui use, pour la cause, de son prestige en s'appuyant sur les référents de sa génération « qui vécut le rêve de la Révolution et ses défaites » (Ramsîs, 1999, p. 97) n'est pas pour rien non plus dans ce succès: "La censure prudente n'a pas pu toucher à l'un de ses principaux films et des plus sérieux, al-Irhâbî (Le terroriste). Le film put lui échapper grâce à la popularité de son héros, à son statut de vedette et à son influence et bénéficia même de l'appui officiel de l'État, de la protection de toutes les salles où il était diffusé et d'une garde particulière affectée à tous ceux qui y avaient participé : producteur, réalisateur et interprètes. » (Abû Shâdî, 1998, p. 137) Le fait que de nombreux ministres, hommes politiques et intellectuels aient répondu à l'invitation d'Imâm et assisté à la première des films où il critique certaines situations sociopolitiques et s'attaque à des questions épineuses, ne fait que confirmer cette tendance.

Ces «rapports de force» qui ont traditionnellement existé entre le pouvoir et le créateur et qui opposaient tout un arsenal de lois et de règlements à des euphémismes, métaphores et symboles, ne sont plus de mise dans ce type de film ( ${ }^{c} \mathrm{Abd}$ al-Fattâh, 1997). Les films de la nouvelle tendance sont fondés sur le message politique direct où le comique se mêle à la critique et se rapproche parfois du burlesque ${ }^{9}$. Les ingrédients de leur succès résident dans une configuration professionnelle particulière: un réalisateur d'une haute compétence technique, un écrivain chevronné et une star jouissant d'une large popularité (Abû Shâdî, 1998, p. 122) et mettant à profit une expérience de comédien de théâtre acquise dans un climat relativement libéral, dans le sillage des changements politiques survenus au lendemain de l'assassinat de Sadate en 1981. Ayant sondé les réactions du public à ses critiques virulentes lancées sur scène, cÂdil Imâm taille ses rôles cinématographiques avec bon sens, à l'aune de ce public, dans des films qui constituent une sorte d'exutoire au "non-dit » propre à des époques différentes (Ramsîs, 1999, p. 96). Un cinéma basé sur le héros « populaire », " modèle » ou « fidèle ", conscience de la collectivité, incarnant les rêves et la réalité de ce public qui, à son tour, s'unit à lui. Un cinéma fondé sur les traditionnels dualismes : peuple/ pouvoir, individu/institution, privation/satiété... et autres dichotomies obsédantes. Pour de nombreux critiques, ses films comptent parmi « les plus réalistes, les plus audacieux et les plus réussis tout en maintenant un bon niveau technique » (al-cAmrî, 1999, p. 75).

En fait, «l'audace » de ces films réside, en partie, en ce qu'ils s'attaquent aux trois tabous ${ }^{10}$ que sont « la religion, le sexe et la politique ». Quoique superficiel, le traitement de ces tabous est apte à " éveiller les sens du spectateur » sans pour cela aller au delà. Les "modèles " humains décrits par le dramaturge sont l'expression caricaturale ou stéréotypée des éléments de ces tabous (al-cAmrî, 1999, p. 78-80) qui se libèrent parfois 
aux dépens d'autres, selon les exigences de la censure et du guichet. Les dénouements, sans être subversifs, divulgateurs ou naïvement heureux, empruntent à la satire ses ressorts, canalisant par procuration le potentiel de révolte populaire. Ces appels à l'enthousiasme qui vont parfois jusqu'à faire jaillir les larmes, et qui sont soutenus par un sourire, marquant un faux espoir, sont le fait du personnage qui, dans le film, symbolise la capacité de rêver et d'espérer - ces objectifs consensuels de l'art noble (alTalmisânî, 1998 ; al-cAmrî, 1999, p. 77-78).

La seconde conjoncture a produit, elle, le film «fabriqué sur mesure pour l'acteur et le public ", et concerne la fin des années quatre-vingt-dix. C'est le film tout à la fois " ami de la censure " et "ami de la famille». Avec le déclin du genre "terrorisme et corruption", lié à l'épuisement du "créneau " et de son interprète, on assiste à l'apparition d'un nouveau genre, soutenu par une nouvelle star (Hinaydî), fabriquée pour l'occasion et qui se plie à ce que le public attendrait d'elle (Sayf al-Dîn, 1999). Ce genre s'illustre par des comédies proches du vaudeville" familial, où aventures et péripéties, anecdotes et traits d'esprit concourent à donner une vision caricaturale de la société. Insérés dans des sketches, ces ingrédients sont par suite épicés d'allusions politiques, que diffusent des personnages qu'on appelle désormais les «nouveaux comiques » : représentatifs d'une époque qui vit à l'heure des ajustements structurels, ils sont pour la plupart jeunes, chômeurs ou marginaux (Sâmî, 1999 ; Yûsuf, 1999b).

31 L'explication du succès que remportent ces films auprès du public se situe, d'après certains critiques, "hors du domaine artistique». Plongeant ses racines dans le contexte social, ce cinéma remplace, à moindre coût pour le spectateur, le théâtre commercial et touristique (celui s'adressant à un public arabe nonégyptien mais que consomment également certains Égyptiens) (Yûsuf, 1999b) et s'il mêle à son aspect divertissant des questions qu'il est difficile d'aborder dans ce type de théâtre, il le fait sur un mode superficiel, espérant gagner le respect et les faveurs du public (Silayha, 1986, p. 175). Le soulèvement des critiques et des intellectuels contre ce type de films est, de manière générale, inversement proportionnel à l'accueil de plus en plus favorable que le public, qui s'y reconnaît, lui réserve (Ramsîs, 1999 ; Yûsuf, 1999b). En fait, il est au diapason d'un nouveau public de spectateurs, essentiellement formé de jeunes : ses histoires banales, prises dans la réalité de la vie de ces jeunes, reprend leur langage, leurs chansons et leur mode de pensée, reflétant l'état psychologique de la jeunesse, ses désillusions et ses rêves les plus simples avec un humour qui, selon ses producteurs, pénètre les cœurs en toute simplicité et naturel (Sayf al-Dîn, 1999; Qatâmish, 1998). Ce nouveau public cherche, selon les critiques, à jouir du moment présent et évalue le film en fonction du plaisir personnel qu'il y trouve. C'est pour lui le moyen de se divertir loin des débats lassants, des grandes causes et des slogans pompeux (Yûsuf, 1999b). Parallèlement aux analyses des « raisons de cette connivence du public avec le cinéma prédominant » et aux critiques relatives au surinvestissement des modalités de sa réception (Saîd, 1999), apparaissent des tentatives pour expliquer le retrait d'un film tel que 'Araq al-balah (La sueur des palmiers). Réalisé par Radwân alKâshif, ce film, qui avait pourtant remporté plusieurs prix internationaux, fut retiré une semaine seulement après sa diffusion en salle. Boudé par les gros distributeurs parce qu'il s'inscrit contre la vague dominante, il est sacrifié sur l'autel des sacrosaintes modalités de réception : n'a-t-on pas parlé, à son sujet, de "l'incapacité du public à saisir le message du créateur et de l'incapacité du créateur à se mettre à la portée du public » (al-Bahrâwî, 1999) ? Une comparaison fut de même établie entre al- 
Masîr (Le Destin) de Youssef Chahine qui ne put couvrir ses frais (18 millions de livres égyptiennes) que grâce à sa diffusion à l'étranger, dans 120 salles en France notamment, et Ismấîliyya râyih gâyy (Ismâcîliyya aller-retour) de Karîm Diyâ' al-Dîn (à l'origine de la vague des films prédominants) et qui, projeté localement en même temps que Le Destin, réalisa 16 millions de livres égyptiennes (£e) de recettes contre un coût relativement modeste (environ $600000 \mathrm{Ee}$ ), coupant l'herbe sous le pieds du Destin (Fathî, 1998 ; cÂtif, 1998).

Qualifiés par leurs producteurs de divertissants, familiaux, propres et décents, ne s'adressant pas aux sens mais respectant les valeurs de la famille égyptienne et devant lesquels ses membres peuvent à nouveau se réunir - habitude perdue depuis longtemps à cause de la vulgarité des productions habituelles (Sayf al-Dîn, 1999 ; Qatâmish, 1998), ces films issus de l'équation guichet + thème banal + allusions politiques neutres (alTalmisânî, 1998, p. 32) anticipent, sur le mode comique, les attentes de nouveaux spectateurs accédant au marché de la consommation. Consacrant une culture appauvrie, issue du contexte social dominant, ces films-caricatures prennent prétexte de problèmes politiques auxquels ils feignent de trouver des solutions. Ainsi, un traitement superficiel est réservé à des thèmes sociaux et historiques liés à la sensibilité collective et populaire, tels que le conflit israélo-arabe ou les rapports de citoyenneté entre individus de confessions différentes (Yûsuf, 1999a et b) ; cependant que la femme y est toujours reléguée à l'arrière-plan, occupant une place marginale dans l'intrigue et se laissant désigner dans le scénario par un ensemble de termes péjoratifs (Yûsuf, 1999b). S'appuyant sur le canevas d'anciennes séries qui ont fait leurs preuves et qui rassemblaient des sketches pouvant subir, sans préjudice pour le déroulement de l'intrigue, suppressions et ajouts, ces films réactualisent de vieilles configurations sociales sous des formes nouvelles, des configurations appartenant parfois à un monde qui existait " avant même la création du cinéma » (Yûsuf, 1999a et b). L'intrigue de ces films et la logique qui préside à leurs dénouements s'appuyant, on l'a déjà dit, sur une note de "faux optimisme ", les traits sociaux de la réalité actuelle y sont exploités pour vendre « un peu de réel et beaucoup de chimères ». S'adressant à un public qui est «à la recherche d'un instant de répit où il peut oublier les soucis insolubles qui l'accablent ", ces films ont sans doute encore de beaux jours devant eux... à moins que le public, à son réveil, ne réalise, avec encore plus d'amertume, qu'il est obligé de les affronter et que ce cinéma n'en riait pas avec lui mais se riait de lui (Yûsuf, 1999b).

«La créativité égyptienne est toujours garante de son propre redressement. » Tels sont les propos du nouveau censeur officiel, c'est-à-dire du nouveau directeur de la Censure sur les œuvres artistiques, en poste depuis le $1^{\text {er }}$ novembre 1999, dans un entretien intitulé «La nouvelle tendance de la censure »". Ces derniers viennent confirmer les positions qu'il avait déjà défendues lors d'un précédent entretien publié sous le titre: "Je prends le parti du créateur "". Lui-même réalisateur de films documentaires, ce censeur refuse de céder à cette nouvelle donne commerciale, artistiquement pauvre et qu'il est difficile de considérer comme porteuse d'une vision cinématographique - à l'instar de ce cinéma parallèle dont nous faisions mention dans notre introduction. Non sans ironie, il ajoute : «Mais après tout, les opérations de tri et de filtrage ne sont-elles pas tributaires d'un esprit critique et ne sont-elles pas garanties par les canaux porteurs de cette critique et par l'existence même d'une critique ? ""

Traduit de l'arabe par Samia Rizk 


\section{BIBLIOGRAPHIE}

‘Abd al-Basîr al-Husayn, 1999, Matâ yuclinûn wafât al-cinema al-masriyya ? (Quand annoncera-t-on la mort du cinéma égyptien ?), al-Kutub weghat nazar, 7 août.

‘Abd al-Fattâh Muhammad, 1997, «al-Cinema wa-l-sulta » (Le cinéma et le pouvoir), Al-funûn, nº 64 .

cAbdû Ghunaym, 1999, « Mazîd min al-intâg yuslih al-cinema » (Une plus grande

production peut réformer le cinéma), al-Kawâkib, 2 mars.

cÂtif Ahmad, 1998, «Succès croisés », Al Ahram Hebdo, 31 déc.-6 janv.

Abû Shâdî ‘Alî, 1998, al-Cinema wa-l-siyâsa (Cinéma et politique), Le Caire, Dâr sharqiyyât li-l-nashr wa-l-tawzî̀.

Al-cImarî Amîr, 1999, Min humûm al-cinema al-carabiyya ilâ cinema al-ru'ya al-dhâtiyya (Des préoccupations du cinéma arabe au cinéma réflexif), Le Caire, al-Hay'a al-câmma li-qusûr althaqâfa.

Al-Bahrâwî Sayyid, 1999, « al-Cinema wa inqitâcal-tawâsul » (Cinéma et rupture), Akhbâr al-adab, 11 juillet. Al-Cinema wa-l-nâs, 1998, nº $637 ; 1999, n^{\circ} 649$.

Al-Kardûsî Mahmûd, 1999, « Cinema al-hawiyya bayna al-tahmîsh wa sinâcat al-ustûra » (Le cinéma identitaire entre marginalisation et légende), Table ronde organisée par cAbdallah Fathî, al-cUsûr al gadîda, $\mathrm{n}^{\circ} 1$, septembre.

Al-Talmisânî May, 1998, « al-Cinema al-misriyya juzûr wa âfâq » (Le cinéma égyptien : ses racines et ses horizons), al-Fann al-sâbic, $n^{\circ} 4$, août.

Bernard-Maugiron Nathalie, 1997, « Anatomie sociologique d'une affaire égyptienne. Le procès de "L’Émigré" de Youssef Chahine », Boëtsch G., Dupret B. \& Ferrié J.-N., Droit et sociétés dans le monde arabe, Presses universitaires d'Aix-Marseille.

Botiveau Bernard, 1993, «Penser, dire et interdire : logiques et enjeux de la censure des écrits en Égypte ", Égypte/Monde arabe, $\mathrm{n}^{\circ} 14$.

Dardîr Intisâr, 2000, « Aflâm sâdarat-hâ al-raqâba wa ahabbat-hâ al-gamâhîr » (Des films saisis par la censure et aimés du public), Akhbâr al-nugûm, 1er janvier.

Darwîsh Dîna, 1999, « Les censeurs : des fonctionnaires comme les autres », Al Ahram Hebdo, 5-11 mai.

Darwîsh Mustafâ, 1995, « al-Raqâba wa-l-cinema al-ukhrâ : shahâdat raqîb » (La censure et l'autre cinéma : témoignage d'un censeur), Alif, majallat al-balâgha al-muqârana, $\mathrm{n}^{\circ} 15$.

Fathî Muhyî al-Dîn, 1998, « Ismâvîliyya râyih gâyy », al-Funûn, automne-hiver.

Fawzî Nâgî, 1997, Nasharât al-cinema fî misr (Les annales cinématographiques égyptiennes), Le Caire, al-Markaz al-qawmî li-l-cinema, coll. « Ittigâhât naqdiyya ».

Galal Dîna, « La restructuration de l'industrie cinématographique égyptienne dans le cadre de la libéralisation économique », El Khawaga D., Kienle E., Structures politiques et logiques d'action face à la libéralisation économique : processus distributifs et normatifs dans les pays arabes de la Méditerranée, Cedej/Freie Universität Berlin, (à paraître). 
Hasan Basant, 1999, «Raqâbat al-tilifisiûn : uqûd idhcân tantahik hurriyyat al-ibdâc" (La censure télévisuelle : des contrats d'allégeance qui violent la liberté de création), al-Fann al-sâbic, novembre.

Hilmî Sharîf :

- 1998a, « al-Tilivisiûn yatasallal ilâ carsh al-cinema » (La télévision se hisse sur le trône du cinéma), al-Fann al-sâbic, février.

- 1998b, « Lâ badîl can cawdat al-film al-rûmânsî̀ (Le retour inéluctable du film romantique), al-Fann al-sâbic, juin.

Karkûtî 'Alâ', 1999, « Aflâm fî sâlât al-card binâ'an calâ talab amrîca » (Des films diffusés

en salle à la demande de l'Amérique), al-Fann al-sâbic, août.

" Mucgizat al-cinema al-îrâniyya » (Le miracle du cinéma iranien), al-Fann al-sâbic, mai 1998.

Mumtâz Ittidâl, 1995, Mudhakkirât raqîba (Mémoires d'un censeur), Le Caire, GEBO.

Qatâmish Pakinam, 1998, «Idhâ kâna Ismấ̂̂liyya râyih gâyy tâfihân, fa marhaban bi-l-

tafâha » (Si le film Ismâ'îliyya aller-retour est futile, alors bienvenue à la futilité), al-Fann al-sâbic, août.

Râghib Nabîl, 1996, Funûn al-adab al-câlamî (Les genres littéraires mondiaux), Le Caire, al-Sharika al-masriyya al-câlamiyya li-l-nashr/Longman.

Ramsîs Amal, 1999, "Qiyâm wa suqût dawlat cÂdil Imâm » (Grandeur et décadence de l'Empire de cÂdil Imâm), Adab wa naqd, mai.

Sacad cÂdil :

- 1998a, « Awlâd Husayn Sidqî ahraqû aflâmahû » (Les enfants de Husayn Sidqî ont brûlé ses films), al-Musawwar, 18 septembre.

- 1998b, « Hal min haqq al-waratha icdâm acmâl al-mubdiîn? " (Les héritiers ont-ils le droit de détruire les œuvres des créateurs ?), al-Musawwar, 18 septembre.

Saîd Sayyid, 1999, "Âliyyât al-calâqa bayna al-mutafarrig wa-l-cinema » (Les modalités du rapport spectateur/cinéma), al-Fann al-sâbic, numéros de janvier et d'avril.

Salâh al-Dîn Muhammad, 1996, al-Cinema wa-l-sulta (Le cinéma et le pouvoir), Le Caire, Madbûlî.

Sâmî Karma, 1999, "Hammâm fî amsterdam : khutwâ saghîrâ ilâ-l-amâm » (Hammâm à Amsterdam : un petit pas en avant), al-Fann al-sâbic, octobre.

Sayf al-Dîn Husâm, 1999, « Al-sinârist Midhat al-cAdl : yakfí annana acadnâ al-gumhûr al-misrî li-lcinema » (Le scénariste Midhat al-cAdl : il nous suffit d'avoir ramené le public égyptien au cinéma), al-Fann al-sâbic, mai.

Shafik Viola, 1998, Arab Cinema: History and Cultural Identity, Le Caire, AUC Press.

Sharaf al-Dîn Durriyya, 1992, al-Cinema wa-l-siyâsa fî misr (Cinéma et politique en Égypte), Le Caire, Dâr al-shurûq.

Silayha Nihâd, 1986, al-Masrah bayna al-fann wa-l-fikr (Le théâtre entre art et pensée), Le Caire, GEBO.

Soueif Mustapha, 2000, Misr al-hâdir wa-l-mustaqbal (L'Égypte : le présent et l'avenir), Le Caire, Kitâb al-hilâl. 
Yûsuf Ahmad :

- 1999a, « al-Cinema al-misriyya tamdî ilâ masîriha al-bâ’is » (Le cinéma égyptien suit son impitoyable destin), Akhbâr al-adab, $n^{\circ} 19$, septembre.

- 1999b, « Hammâm wa-l-mudhikûn al-gudud : cafwiyya muzayyafa tahtaqir al-mar'a wa tastaghill humûm al-gumhûr » (Hammâm et les nouveaux comiques : une fausse naïveté qui méprise la femme et exploite les soucis du public), Akhbâr al-adab, 3 octobre.

Zuhayrî cAmr, 1999, « Génération Heneidî », Al Ahram Hebdo, 27 janvier-2 février.

\section{NOTES}

1.Al-Musawwar,17/12/1999, entretien avec Madkûr Thâbit, propos recueillis par cAbd alNûr Khalîl.

2.Adab wa naqd, $\mathrm{n}^{\circ}$ 161, janvier 1999, entretien avec le réalisateur cÂtif al-Tayyib, propos recueillis par Fatma Tufaylî.

3.Al Ahram Hebdo, 5-11/05/1999, entretien avec cAlî Abû Shâdî, réalisé par Dina Darwîsh. 4.Al Ahram Hebdo, 25/11-01/12/1999, entretien avec cAlî Abû Shâdî, réalisé par Soheir Rahmî et Ahmad cÂtif.

5.Al-Fann al-sâbic, « Le miracle du cinéma iranien », mai 1998, p. 60-63.

6.Al Ahram Hebdo, 25/11-01/12/1999, entretien avec cAlî Abû Shâdî, réalisé par Soheir Rahmî et Ahmad cÂtif.

7.Al Ahram Hebdo, 5-11/05/1999, et l'entretien avec 'Alî Abû Shâdî, réalisé par Dina Darwîsh.

8.La loi $n^{\circ} 3$ de 1996 (suivie de l'amendement à l'article 3 du Code de procédure civile), stipule que seul le Parquet peut désormais se porter requêrant en matière de hisba, ce qui abolit virtuellement la hisba du droit égyptien.

9.En français dans le texte.

10.Idem.

11.En français dans le texte.

12.Al-Ahrâm, 26/01/2000, Entretien avec Madkûr Thâbit, propos recueillis par Muhammad Nasr.

13.Al-Musawwar, 17/11/1999, Entretien avec Madkûr Thâbit, propos recueillis par cAbd al-Nûr Khalîl.

14.Idem.

\section{INDEX}

Mots-clés : cinéma, censure 